\title{
FORTUNA Y GLORIA. UNA LECTURA HISTÓRICA Y ARQUEOLÓGICA DEL UNIVERSO INDIANA JONES
}

\author{
David Quixal Santos \\ Universitat de València
}

\begin{abstract}
Resumen: En el presente trabajo se pretende abordar los escenarios, características y temáticas de las diferentes obras de la saga Indiana Jones. La base principal será la archiconocida trilogía de películas originales, pero también se manejarán otro tipo de formatos dentro de este profuso y diversificado universo de ficción. En primer lugar, se analizarán en clave histórica y arqueológica los yacimientos, culturas y materiales aparecidos en estas producciones. Posteriormente, el ciclo nos servirá para evaluar la percepción que la sociedad desde finales de siglo XX ha tenido de la disciplina arqueológica y cómo ésta queda reflejada en la cultura de masas.
\end{abstract}

Palabras clave: Indiana Jones, Arqueología, yacimientos arqueológicos, cine de aventuras, aventura gráfica.

\section{Fortune and Glory. An historical and archaeological reading of the Indiana Jones Universe}

Abstract: In this paper, I approach to the scenarios, features and topics from the different works of the Indiana Jones saga. The main base will be the well-known trilogy of original films, but other formats will also be managed within this profuse and diversified universe of fiction. Firstly, I will analyze the sites, cultures and materials which appear in these productions from an historical and archaeological view. Subsequently, the cycle will help us to see the perception of archaeological discipline that society has had since the end of the $20^{\text {th }}$ century and how it is reflected in the mass culture.

Key words: Indiana Jones, Archaeology, archaeological sites, adventure movies, graphic adventure.

\section{INTRODUCCIÓN AL PERSONAJE Y LA SAGA}

En 1981 se estrenó Raiders of the Lost Ark, la primera de las tres películas que componen la trilogía inicial de aventuras del arqueólogo Indiana Jones (fig. 1.1). En ese momento sus creadores no podían sospechar el alcance que iba a tener la misma, tanto en la propia configuración del cine de aventuras como en la historia del cine en general. Detrás de esta tríada fílmica también encontrábamos un destacado tridente cinematográfico: la conjunción de George Lucas (American Graffiti, 1973 y Star Wars, 1977, entre otros), como creador y pro-

Data de recepció: 20 de maig de 2019 / Data d'acceptació: 31 de maig de 2019 . 

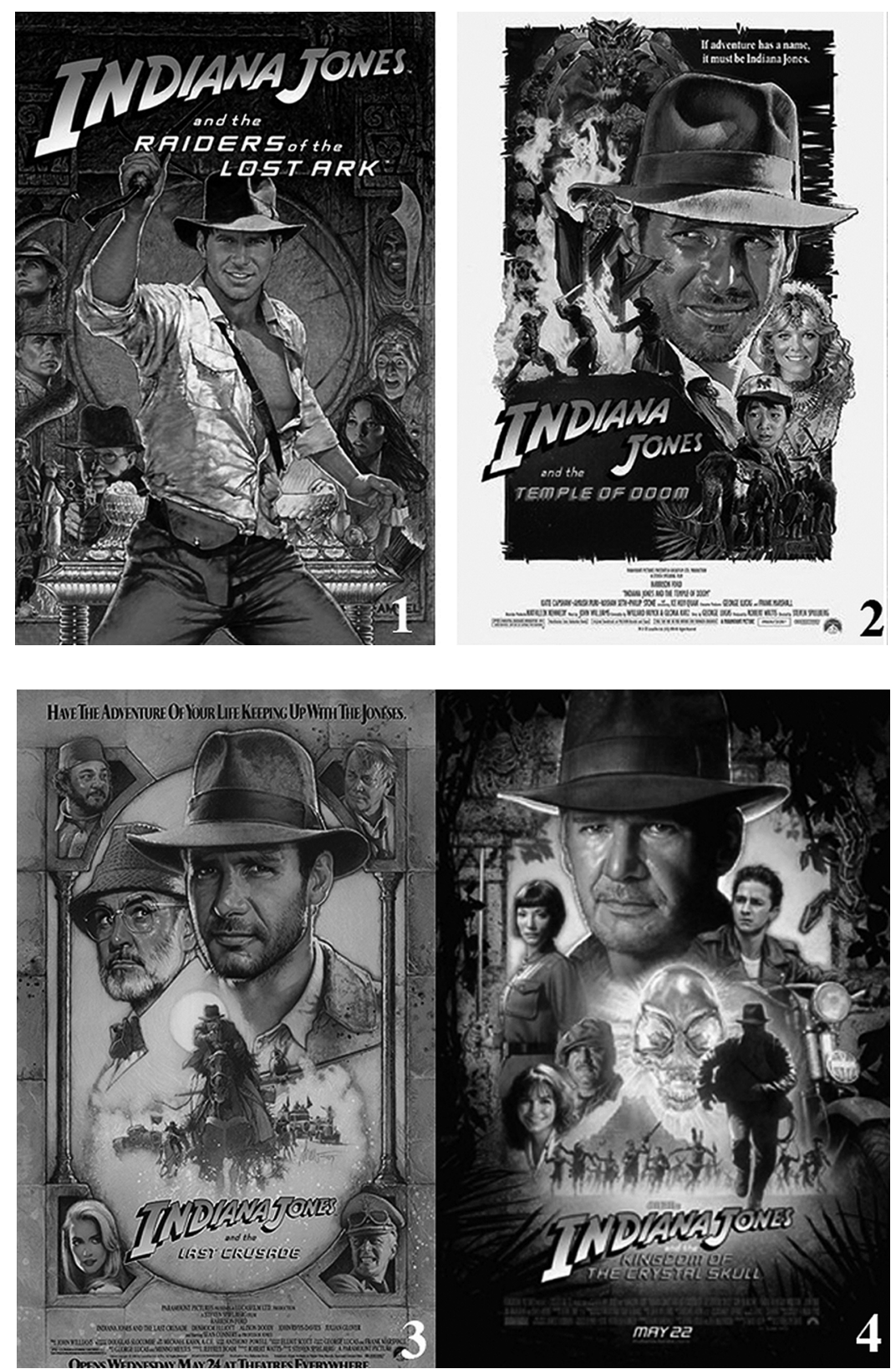

Figura 1. Carteles de las películas Raiders of the Lost Ark (1), Indiana Jones and the Temple of Doom (2), Indiana Jones and the Last Crusade (3) e Indiana Jones and the Kingdom of the Crystal Skull (4). 
ductor; Steven Spielberg (Jaws, 1975, E.T., 1982 o Schindler's List, 1993, entre otros), en la dirección; y Harrison Ford (Han Solo en Star Wars, 1977 y Rick Deckard en Blade Runner, 1982, entre otros), de actor principal (fig. 2). Lawrence Kasdan, guionista de esta primera película, también fue clave en la creación del personaje. En 1984 siguió la controvertida Indiana Jones and the Temple of Doom (fig. 1.2) y en 1989 la aclamada Indiana Jones and the Last Crusade (fig. 1.3). En 2008 se sumó una esperada cuarta entrega, Indiana Jones and the Kingdom of the Crystal Skull (fig. 1.4), cuyas críticas recibidas la situaron muy lejos del éxito de los años 80. Todas ellas, producidas por Lucasfilm Ltd., distribuidas por Paramount Pictures y con una aclamada banda sonora compuesta por John Williams, han dado a luz a un personaje fundamental en la historia del celuloide. Por último, cabe citar que en 2020 se tiene previsto el inicio del rodaje de una quinta entrega, que llegará a las salas en 2021.

Tal y como en repetidas ocasiones han indicado sus propios creadores, el personaje no surgió ex nihilo. Sus aventuras siguen el esquema típico de toda producción épica, desde las epopeyas heroicas a la literatura exótica y colonial (González, 2011, 53). La figura de este arqueólogo / héroe aventurero bebe de las miniseries de los años 30 y 40, así como de toda la literatura pulp (libros pequeños de formato barato) y cómics de primera mitad del s. XX, con personajes como The Lone Ranger o Flash Gordon. A su vez, algunos filmes de aventuras de mediados de siglo pasado aportaron la estética al personaje principal, véase The Treasure of the Sierra Madre (Huston, 1948), King Solomon's Mines (Bennett y Marton, 1950), The Secret of the Incas (Hopper, 1954) o Valley of the Kings (Pirosh, 1954). El resultado es un protagonista que ejerce como arqueólogo, pero tiene tintes de soldado, espía, detective y erudito.

No obstante, en la retina de sus productores y guionistas sin duda estaban todos los grandes descubrimientos arqueológicos de la horquilla comprendida entre mediados del siglo XIX y primer tercio del XX. Hallazgos en los albores de la Arqueología como disciplina, siempre cargados de ciertos tintes épicos. Los materiales, las historias y la fotografía que nos han legado han configurado la imagen romántica que la sociedad occidental ha tenido de la recuperación de restos arqueológicos. En este sentido, tenemos conocidas muestras de Arqueología filológica, en las que arqueólogos como Heinrich Schliemann buscaban localizar enclaves del ciclo homérico como Troya (1871) o Micenas (1874). Arthur Evans en Creta emprendió la labor de localizar el palacio de Cnosos que daba lugar a la leyenda del rey Minos y el Minotauro (1900). También podemos sumar los descubrimientos en lugares exóticos e inhóspitos, bien sea en selvas frondosas como Machu Pichu (Hiram Bingham en 1911), bien en medio del desierto como Nínive (Austen Henry Layard en 1847) o Babilonia (Robert Koldewey en 1899). En la mayoría de los casos no se trataba de descubrimientos directos, simple- 
mente expediciones occidentales que localizaban enclaves de sobra conocidos por la población autóctona. En otros, los protagonistas daban comienzo a las primeras intervenciones arqueológicas en los yacimientos. Fundamentales eran aquellos descubrimientos que han entrado en el Olimpo de la Arqueología por la riqueza y estado de conservación de sus materiales, siendo especialmente conocido el hallazgo de la cámara mortuoria de Tutankamón (Howard Carter en 1922). La prueba de que todas estas estampas de Arqueología decimonónica y de principios del XX estaban presentes en la gestación de las producciones es la elección de la ciudad egipcia de Tanis, la "Tebas del Norte", como escenario principal de la primera película. De Tanis investigadores como William M. Flinders Petrie, Auguste Mariette o Pierre Montet nos ha legado un excepcional corpus documental y fotográfico (Brissaud y Zivie-Coche, 2000).

Son abundantes las referencias, artículos y reportajes que tanto la literatura histórica como cinematográfica han dedicado a intentar descubrir el "verdadero" Indiana, el personaje real que pudo servir de inspiración para la creación del protagonista y la saga (Bonn-Muller y Powell, 2008; Gresh y Weinberg, 2008, 27-33). Las propuestas son múltiples y muy variopintas. Sin embargo, pensamos que más que tratarse de un personaje en concreto, la clave está en el citado corpus de descubridores y descubrimientos, que ha enraizado en el imaginario colectivo. Hechos y personajes reales e inventados se entrelazan constantemente, siendo complicado para el gran público saber discernirlos.

George Lucas era un amante de la Historia y la antigüedad, y todo ello queda reflejado en algunas de sus producciones. La aparición del arqueólogo americano y sus aventuras volvía a avivar este interés por lo antiguo, el ligamen entre los descubrimientos arqueológicos y el mundo de ficción. No obstante, no era algo nuevo. A finales del siglo XIX, de forma paralela a muchos de los descubrimientos anteriormente citados, surgió el género literario Lost world a partir de la obra de Henry Rider Haggard King Salomon's Mines (1885), fruto del fervor suscitado y su eco en la prensa.

Del mismo modo, si el listado de influencias en la configuración de este universo es extenso, también lo es el abanico de influjos que este ha generado en producciones cinematográficas o literarias posteriores, bien sea por el tipo de aventuras desarrolladas, bien por el entusiasmo suscitado en torno al mundo de la Arqueología. Así tenemos películas como Romancing the Stone (Zemeckis, 1984), King Salomon's Mines (J. Lee Thompson, 1985), The Mummy y The Mummy Returns (Sommers, 1999 y 2001) o National Treasure (Turteltaub, 2004). La serie de videojuegos protagonizados por la arqueóloga Lara Croft: Tomb Raider ha dado lugar a tres películas (West, 2001; De Bont, 2003; Uthaug, 2018), así como las recientes películas de animación de Tadeo Jones (Gato, 2012; Gato y Alonso, 2017), parodia del arqueólogo americano. 


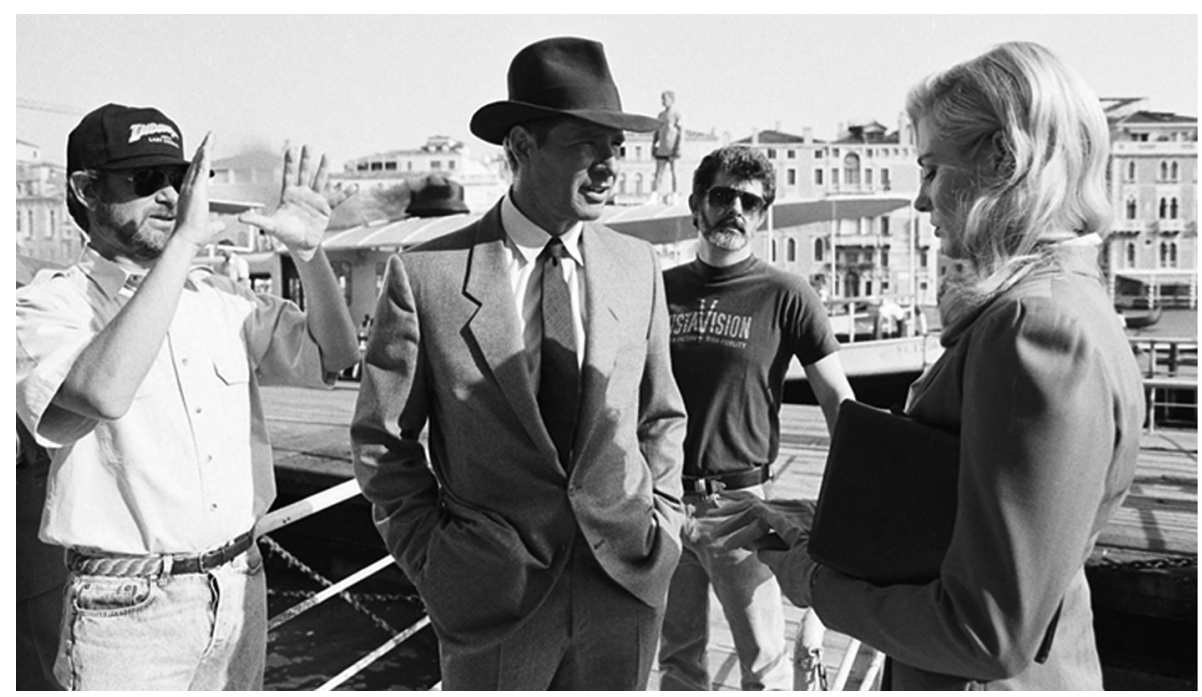

Figura 2. Rodaje de Indiana Jones and the Last Crusade en Venecia en agosto de 1988. De izquierda a derecha: Steven Spielberg (director), Harrison Ford (actor principal como Indiana), George Lucas (productor) y Alison Doody (actriz, papel de Elsa Schneider).

Aunque a lo largo del presente trabajo la base sea siempre la saga de películas, la franquicia de Indiana Jones ha aumentado exponencialmente a lo largo de las tres últimas décadas, diversificándose en series, novelas, cómics, videojuegos y un largo etcétera (Luceno, 2008). Merece una mención especial la serie de televisión The Young Indiana Jones Chronicles (1992-1996).

\section{UNA MEZCLA DE REALIDAD \& FICCIÓN AL GUSTO DEL ESPECTADOR}

No pretendemos en estas líneas desprestigiar el género cinematográfico, con su particular lenguaje narrativo o capacidad creativa (Bordwell, 1996). Sobra decir que se trata de películas y que, por lo tanto, sus creadores dan preponderancia a la trama por encima del realismo. Todo filme es un filme de ficción (Aumont et al., 1985, 100). Aunque puedan estar basadas en cuestiones, objetos o yacimientos existentes, el rigor histórico queda en un lugar secundario en detrimento del guion. Productores y guionistas buscan despertar un interés y atracción por lo antiguo, por lo inexplorado, por lo desconocido; y para ello modelan unas historias en las que la tónica es una constante fusión de ele- 
mentos reales e inventados, dando lugar a unos mundos imaginados con características híbridas, tanto en lo artístico como en lo cultural. Al presentarse como una historia, el filme adquiere un valor esencial: ser como la realidad, imprevisible y sorprendente. La apariencia de "verdad" resultante de esa diégesis es lo que permite a los productores enmascarar todo lo arbitrario del relato y la constante interacción entre narración y estereotipos (Aumont et al., 1985, 121). Existen ya diversos e interesantes trabajos en los que se realiza un análisis de la totalidad de elementos históricos que aparecen en las películas, para determinar qué hay de verosímil y qué de imaginado en cada una de ellas (Gresh y Weinberg, 2008; Cano, 2017). Para un conocimiento más extenso remitimos a los mismos, ya que en el presente artículo el interés radica más en el sentido de la propia fusión que en los hechos en sí.

Los propios creadores defienden su elaboración de MacGuffins (término acuñado por Alfred Hitchcock), objetos que sirven para poner en marcha la acción, pero que en ocasiones son irrelevantes en el desarrollo de la trama (VVAA, 2008, 12). El Arca de la Alianza en Raiders of the Lost Ark, las Piedras de Sankara en The Temple of Doom o el Santo Grial en The Last Crusade acaban siendo lo de menos en los respectivos argumentos. Se hubiese podido escoger otro objeto de igual antigüedad y semejantes características y las tramas podrían haber sido exactamente las mismas. Son elementos codiciados porque otorgan poder, riqueza o vida eterna y su búsqueda nos recuerda a la leyenda de Jasón y el Vellocino de Oro de la mitología griega (De la Plaza, 2016, 202203). Su valor no sólo radica en el objeto, sino también en lo que aporta a nivel interior la propia búsqueda en sí.

En Raiders of the Lost Ark la acción nos remite, tal y como hemos indicado anteriormente, a las arenas de Egipto, a Tanis, una ciudad que cobra importancia durante la Baja Época como sede de las dinastías XXI y XXII. El MacGuffin de esta película es la mítica Arca de la Alianza, objeto que custodiaba las tablas de la ley desde el Éxodo, supuestamente albergado en Jerusalén en el Tabernáculo y posteriormente ya dentro del primer templo de Yahvé construido por Salomón. A este objeto se le pierde la pista a comienzos del s. VI a.C. tras el saqueo de Jerusalén por las tropas de Nabucodonosor II y el consiguiente traslado de reliquias a Babilonia (II Reyes, 24:10-16). La elección de Tanis como sede para el Arca viene dada a partir de la mención en la película a Shishaq, rey de Egipto aparecido en los textos bíblicos y que supuestamente atacó Jerusalén (I Reyes, 14:25-26 y II Crónicas, 12:9). Este rey se asocia con Sheshonq I, primer faraón de la dinastía XXII (3er Periodo Intermedio) y que tenía su sede en Bubastis (Shaw, 2007, 438). Sin embargo, la referencia en el filme es anacrónica puesto que la acción tuvo lugar durante la segunda mitad del s. X a.C. (Liverani, 2005, 120-123), mientras que hay textos bíblicos que 
indicarían la permanencia de las reliquias en Jerusalén hasta la expansión neobabilónica (II Crónicas, 35:3; II Reyes, 23:21; II Macabeos, 2:4-5). Del mismo modo, tampoco se puede sostener la presencia en Tanis del llamado Pozo de las Almas, lugar que la tradición ubica en Jerusalén, concretamente debajo de la Cúpula de la Roca. En la tradición hebrea es el lugar donde Abraham iba a sacrificar a su hijo Isaac por orden de Dios (Génesis, 22), mientras que para el mundo musulmán es desde donde el profeta Mahoma ascendió a los cielos. Otro de los objetos fundamentales de la trama es el llamado "Bastón de Ra", pieza crucial para localizar el enclave del Arca (fig. 3.2). En ella encontramos una peculiar fusión iconográfica, al estar inspirado tanto en las joyas halladas dentro de la tumba del faraón Tutankamón como en blasones heráldicos del Japón medieval (Cano, 2017, 41-46).

En The Temple of Doom el argumento transcurre en la India. El escenario principal lo constituye un ficticio palacio de Pankot que se tuvo que ambientar en los desaparecidos estudios Elstree de Inglaterra (fig. 3.5). Inicialmente se pretendía rodar en el Fuerte Amber y el Palacio de los Vientos de Jaipur (Rajastán, India), pero la posibilidad les fue denegada, entre otras cosas por las diferentes escenas en las que el pueblo hindú se veía ridiculizado, como la controvertida escena del banquete. Por debajo del palacio se ubica un antiguo templo de la secta Thuggee (derivado de Thag, ladrón en lengua hindú), fraternidades que ya habían aparecido en otras películas anteriores como Gunga Din (Stevens, 1939). Esta secta existió realmente, eran conocidos como "los estranguladores" y pulularon por caminos y zonas rurales de la India desde el medievo hasta época colonial británica. Se dedicaban a asaltar a viajantes, robarles y ejecutarles en honor a la diosa Kali, símbolo de lo maligno y de la muerte, puesto que cada muerte suponía atrasar un milenio el regreso de la diosa. No obstante, la imagen que nos ha llegado parece que también responde a las exageraciones derivadas del imaginario colonial británico tendentes a desprestigiar a la sociedad hindú (Van Woerkens, 2002). En esta misma línea, en la película se les atribuyen determinados tipos de sacrificios que pertenecen a otras tradiciones culturales, como las extracciones del corazón en vida (ámbito mesoamericano), beber sangre (simbología tibetana), arrojar los cuerpos a lava ardiendo (mundo polinesio) o el empleo de muñecos vudú (ámbito africano). Todo con la finalidad de exagerar el argumento y la trama de la película.

En la tercera entrega, The Last Crusade, se utilizan escenarios históricos como la Iglesia de San Moisè en Venecia, reconvertida en una biblioteca. El MacGuffin es el Santo Grial, el cáliz que según la tradición bíblica Jesús utilizó en la Última Cena y con el que José de Arimatea recogería su sangre tras la crucifixión. José de Arimatea, encargado de recuperar el cuerpo de Jesús y darle sepulcro, sería el encargado de llevar la reliquia a Inglaterra después de la 

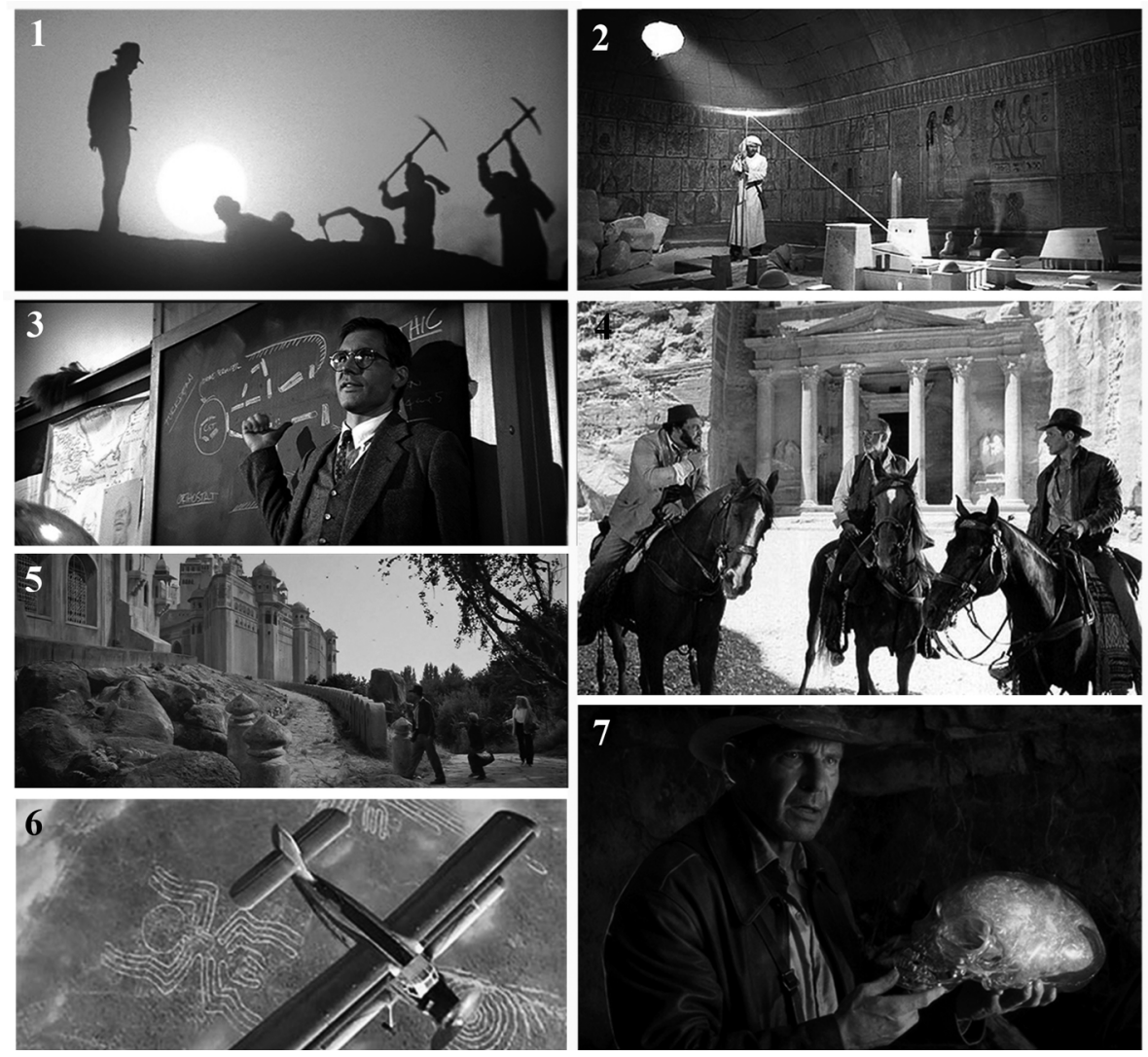

Figura 3. Fotogramas de las películas Raiders of the Lost Ark (1, 2 y 3), Indiana Jones and the Temple of Doom (5), Indiana Jones and the Last Crusade (4) e Indiana Jones and the Kingdom of the Crystal Skull (6 y 7).

resurrección. Vemos como la pieza, per se, ya constituye un auténtico palimpsesto literario e histórico, donde a las escasas menciones a una copa en los evangelios (Mateo, 26:27-29; Lucas, 22:17-20) se fueron añadiendo características legendarias a lo largo de diferentes relatos medievales. Especialmente cuando a finales del siglo XII el autor francés Robert de Boron fusionó las informaciones bíblicas con leyendas artúricas, a las que posteriormente se añadió todo el imaginario templario (Cano, 2017, 73-79). En la película el cáliz se alberga en un templo cruzado cerca de Alejandreta, antigua fundación de Alejandro Magno próximo al punto donde tuvo lugar la histórica batalla de Issos (Alexandra ad Issum). Esta ciudad actualmente se encuentra en Turquía, pero 
en el 1938 en que transcurre la acción correspondería a la República de Hatay (Iskenderun). Para emular el santuario se utiliza como decorado real el templo nabateo rupestre del Tesoro de Petra (Jordania) (fig. 3.4), algo incoherente a nivel histórico o arquitectónico.

Esta dinámica híbrida tiene continuidad en la secuela, The Kingdom of the Crystal Skull, donde se utiliza como MacGuffin determinadas calaveras de cristal (fig. 3.7). Estas piezas, presentes en diferentes colecciones (The British Museum o el Smithsonian), han generado mucha controversia ya que se les pretendía otorgar una cronología precolombina (Mitchell-Hedges, 1954), algo que no entroncaba con sus técnicas de fabricación. Recientemente se ha demostrado por investigadores británicos que se trata de falsificaciones u objetos litúrgicos decimonónicos realizados en cristal de cuarzo (Walsh, 2005, 2008 y 2010). La película nos muestra una auténtica amalgama de culturas precolombinas donde se fusionan las Líneas de Nazca (fig. 3.6), la Leyenda del Dorado (Akator) y construcciones piramidales (típicas de Mesoamérica) en plena selva amazónica. Por no hablar de que en última instancia todo está ligado a una civilización superior extraterrestre...

Por último, sacamos a colación un elemento del ciclo Indiana Jones no tan conocido, pero que refleja muy bien las mismas características que las películas, precisamente porque su trama surge de un guion descartado. El videojuego Indiana Jones and the Fate of Atlantis (1992), desarrollado por LucasArts (compañía filial de George Lucas), se convirtió en los años 90 en una de las aventuras gráficas más exitosas (fig. 4.1). En este tipo de juegos es fundamental pensar para poder avanzar, mediante la resolución de rompecabezas, la interacción con personajes o la búsqueda y uso de determinados objetos. El binomio que significaba aunar una aventura gráfica con el universo Indiana Jones, famoso por despertar ese anhelo por los descubrimientos y generar atracción por la Antigüedad, llevó al éxito del videojuego.

En el mismo volvemos a encontrar esta fusión de elementos, en este caso con la excusa de la búsqueda del mítico continente de la Atlántida. Descrita por Platón en sus Diálogos Timeo y Critias, constituyó una utopía en la que se proyectaban ideales y valores de Atenas, otras poleis griegas y Oriente (Durán y Lisi, 1992, 268-271). Su supuesta ubicación ha sido planteada con mayor o menor rigurosidad en infinidad de lugares (Santorini, Doñana, Azores, Canarias, América, etc.) (Barceló, 1998), algunos de los cuales aparecen recogidos en el videojuego. El final de la Atlántida llegaría "tras un violento terremoto y un diluvio extraordinario, en un día y una noche terribles, (...) la isla de la Atlántida desapareció hundiéndose en el mar" (Timeo, 25). También aparece toda la simbología mítica tradicionalmente asociada a la civilización atlante como discos de piedra (imitando la supuesta trama urbana en diferentes ani- 
llos de tierra - fig. 4.6) y el orichalcum, metal que "se extraía de la tierra en muchos lugares de la isla, el más valioso de todos los metales de entonces, con la excepción del oro" (Critias, 114). Este material se ha relacionado con el llamado "latón dorado" una aleación de cobre, zinc y plomo que encontramos en conjuntos arqueológicos como el pecio de Gela (Sicilia) (Forssmann, 2017). También se ha interpretado como una posible alusión al ámbar báltico, pese a no tratarse de un metal. En el videojuego se le dota de propiedades especiales, siendo capaz de poner en funcionamiento objetos, máquinas y armas, motivo por el que los nazis andan tras su búsqueda (fig. 4.3).

De nuevo, la trama no se circunscribe exclusivamente al reino perdido, sino que encontramos conexiones con otras culturas y regiones: templo maya de Tikal (fig. 4.2), excavaciones en Argelia (fig. 4.5), la Creta minoica... Aparece como uno de los escenarios el yacimiento de Cnosos, "sede" simbólica del laberinto del Minotauro, con los famosos cuernos de la consagración (fig. 4.4). Finalmente, la antigua isla se encuentra sumergida en pleno mar Egeo, enlazando con la teoría que defiende que su ubicación correspondería con la isla de Thera, actual Santorini, colapsada por una erupción volcánica a mediados del II milenio a.C. De hecho, en el juego se menciona a Spyridion Marinatos, excavador del famoso yacimiento de Akrotiri e impulsor de la teoría que vincula la destrucción de la isla de Santorini con el final de la talasocracia cretense (Marinatos, 1939; Marinatos, 2015), si bien la cuestión de la Atlántida siempre quedó al margen.

\section{¿ARQUEÓlOGO, CAZATESOROS O EXPOLIADOR?}

Una de las críticas que siempre ha sobrevolado a este personaje es que no representa la auténtica actividad de los arqueólogos, sino que simplemente se trata de un cazatesoros, figura próxima al coleccionismo de antigüedades (Carvajal et al., 2011). En casi todos los relatos aparecen otros profesionales que se han corrompido y han acabado engrosando las filas enemigas. Por ejemplo, René Belloq, arqueólogo bien formado en la Sorbona, que acaba corrompiéndose y convirtiéndose en expoliador y mercenario al servicio de los nazis en Raiders of the Lost Ark. Sin embargo, los métodos y formas de Indiana y Belloq no dejan de ser bastante similares.

El nacionalsocialismo alemán es el rival por antonomasia en el universo Indiana (Gresh y Weinberg, 2008, 35-37). En todo momento está presente la idea de la Deutsches Ahnenerbe, la sociedad creada por Hitler en 1935 dentro de las SS y liderada por Himmler, cuya finalidad era investigar el ocultismo e intentar recuperar antigüedades y reliquias que pudiesen comportar pruebas de la supe- 

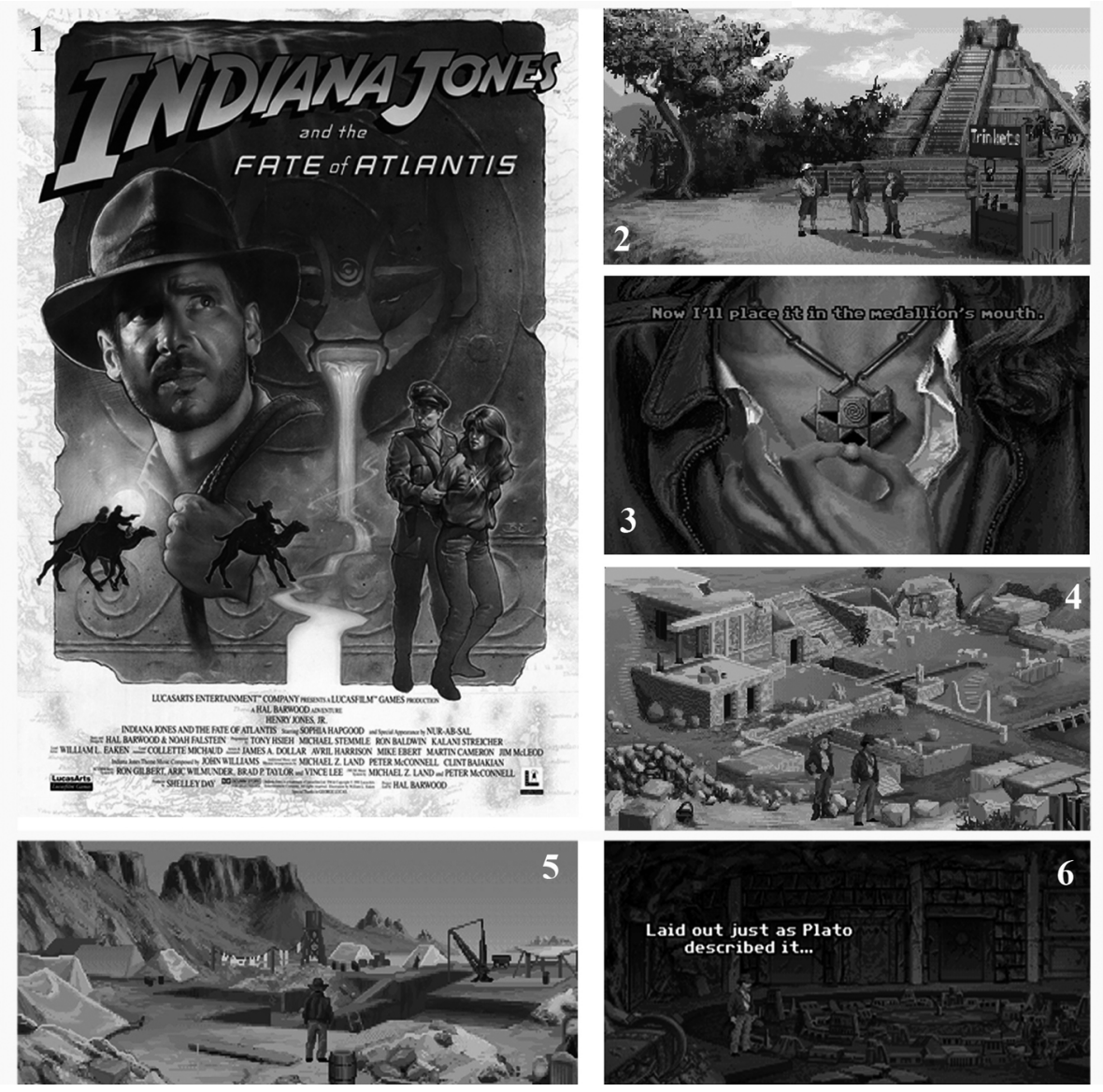

Figura 4. Portada (1) y diversas pantallas (2 a 6) de la aventura gráfica Indiana Jones and the Fate of Atlantis.

rioridad racial aria y justificar la expansión militar alemana (Szczepański, 2009). Incluía más de 40 secciones de diversas disciplinas, ciencias y pseudociencias, llegando a realizar estudios y experimentos con prisioneros de campos de concentración (Härke, 2014). Llevaron a cabo más de 20 excavaciones por Europa, en aquellas zonas y países ocupados militarmente. En esta institución participaron investigadores como Otto W. Rahn, cuya figura sin duda influyó en la configuración de determinados personajes del ciclo, dado su interés en objetos como el propio Santo Grial (Rahn, 1982). Con el cambio de contexto de la cuarta entrega, pasando ya a los años 50 y la Guerra Fría, se introduce en escena a los soviéticos 
que, al igual que los alemanes en las anteriores películas, están cargados de estereotipos (Azcona y Avilés, 2013, 155).

El modus operandi de unos y otros acaba generando en el espectador la peligrosa impresión de que lo verdaderamente importante es el objeto (tendencia compartida con anticuarios, excavadores clandestinos y detectoristas), mucho más que el contexto o el yacimiento, que muchas veces es destruido por obtener la pieza. Paradigmática es la escena en las Catacumbas de Venecia en The Last Crusade en la que Indiana no duda en destrozar una tumba para utilizar un fémur humano a modo de antorcha. El objeto, el tesoro, se antepone al yacimiento (Hall, 2004, 164-167). Cabe recordar como en muchas de las escenas en las que se recupera una pieza valiosa, se hace en detrimento de destruir el contexto (templo del ídolo chachapoyano, templo thuggee, santuario del Santo Grial, tumbas precolombinas...). En muchos casos parece que los personajes realizan una búsqueda a nivel interior, mística, y no tanto arqueológica. En The Temple of Doom, Indiana prefiere dejar la piedra de Sankara en el pueblo al que pertenece antes que darla a conocer o llevarla a un museo.

Estas y otras obras de ficción muestran la labor del arqueólogo y la dan a conocer, aumentando su popularidad, pero por cuestiones de narrativa la elevan a una escala y un tempo muy alejado de la realidad (Ruiz Zapatero y Fernández, 1997). El propio Henry Jones (Sean Connery), padre de Indiana en The Last Crusade, quien representa el modelo de académico tradicional, cuestiona los métodos de su hijo: “¿A esto le llamas Arqueología?”. La visión académica del Dr. Jones queda reducida a las escasas escenas en las que aparece en ficticias universidades (fig. 3.3), con renombradas citas como "La Arqueología busca el hecho, no la verdad. Si es la verdad lo que les interesa, el doctor Tilly imparte filosofía en la clase del fondo"; "Una X no marca el camino"; o "El $70 \%$ de la Arqueología se hace en la biblioteca, investigando, leyendo". Las películas no sólo nos sitúan en el contexto histórico de los descubrimientos que se realizan, sino que también asistimos a la evolución de los conocimientos en Arqueología. En las aulas le vemos hablar sobre el Neolítico, el Megalitismo, las excavaciones de Naucratis o, en la última entrega, aportar una aplaudida cita al arqueólogo marxista Vere Gordon Childe. Por tanto, poco más que leves pinceladas del día a día de esta profesión.

En un momento, décadas de los 80 y 90 del siglo pasado, en el que justamente hace aparición la visión postcolonialista de la Arqueología dentro del Postprocesualismo, el ciclo peca todavía de tener una visión colonialista tanto del pasado como del trabajo arqueológico (recuérdese las escenas de excavaciones en Tanis en Raiders of the Lost Ark, fig. 3.1), juntamente con una visión de supremacía occidental. Esta visión colonial también aparece en la forma de representar las sociedades, un reflejo del imperialismo cultural occidental siem- 
pre presente en Hollywood (Shohat y Stam, 1994). Del mismo modo, tal y como se trató con motivo de la exposición de la Facultat de Geografia i Història de la Universitat de València, Desmuntant Lara Croft, las mujeres tienen un peso secundario en estas películas (Vizcaíno, 2014). Ya sean arqueólogas o no, actúan de forma complementaria, interactuando con humor con el protagonista, a modo de screwball comedy de los años 30-40 del siglo pasado.

En todo momento la Arqueología se representa como una labor individualista, con muy poco trabajo en equipo, cuando la realidad nos muestra precisamente una ciencia donde prima la multidisciplinariedad. Otro aspecto llamativo es el carácter polifacético de Indiana y sus conocimientos sobre prácticamente todo tipo de ámbito cultural: lenguas vivas y muertas, materiales procedentes de periodos culturales diversos, etc. La figura del Dr. Jones choca de bruces con la marcada tendencia hacia una mayor especialización temática y cronológica, especialmente derivada de las propias dinámicas de los estudios doctorales.

A pesar del componente científico de esta profesión, siempre está cargada de tintes aventureros y buscatesoros, que lleva a malinterpretar el método y generar unas expectativas diferentes en cuanto a rigurosidad y resultados. En las películas, los personajes son estereotipos muy marcados, desde el arqueólogo aventurero, al erudito rata de biblioteca, pasando por el arqueólogo corrompido. No obstante, es imposible negar cómo todos estos ciclos de ficción han generado atracción por la disciplina (Hall, 2004, 172), un crecimiento del interés por la antigüedad y los descubrimientos y, hasta un cierto punto, reconocimiento por parte de la sociedad. La vertiente menos espectacular es la que menos interesa a la ficción, pero no es algo muy diferente a lo que pueda suceder con otras profesiones plasmadas en diferentes producciones cinematográficas o televisivas.

\section{CONCLUSIONES}

Somos conscientes de la gran influencia que este ciclo, cargado de estereotipos, metodologías alejadas de la realidad y collages culturales, ha tenido y tiene en la visión de la sociedad de la profesión del arqueólogo. Tal y como hemos visto, en las diégesis de este universo asistimos a una mezcla de diferentes escenarios tanto reales como inventados, en una siempre peculiar fusión de elementos geográficos, culturas históricas y datos arqueológicos bien documentados, juntamente con espacios y sociedades creadas a partir de la imaginación de los propios productores y guionistas. Las películas generan viajes con características místicas, tanto a los protagonistas como a los propios espectadores, tras la búsqueda de objetos mágicos (Macguffins), ocultos en mun- 
dos inhóspitos y desconocidos, cuya recuperación es necesaria para volver al equilibrio original (Arango, 2008). Aspectos esenciales para que esta saga arraigase tanto en el imaginario de varias generaciones en torno a los años 8090. Prueba de este fervor con tintes "retro" es la creación por parte de aficionados de videojuegos como Indiana Jones and the Fountain of Youth, secuelas de Indiana Jones and the fate of Atlantis respetando el diseño, gráficos y jugabilidad originales.

En las obras analizadas es complicado identificar la rigurosa metodología que esta disciplina sigue realmente, ya que las acciones recuerdan más a un simple coleccionista o "buscatesoros". No obstante, sin dejar de ser crítico con este enfoque, tampoco hay que negar el influjo positivo que la trilogía original tuvo a la hora de acercar la Arqueología a un público más amplio, generar cierta simpatía y reconocimiento hacia el gremio, así como despertar un interés por el conocimiento de civilizaciones antiguas entre la cultura de masas. La percepción no debe caer en el catastrofismo y hay que reconocer la importancia que ha tenido y tiene este ciclo de ficción. Prueba de este hecho es que entre 2011 y 2016 viajó por Canadá, EEUU y España la exposición itinerante Indiana Jones and the Adventure of Archaeology, promovida por Lucasfilm y la National Geographic Society, donde se fusionaban objetos procedentes de los rodajes, juntamente con piezas arqueológicas reales y réplicas fidedignas. La exposición llegó a la ciudad de Valencia en el año 2011. En la misma línea, desde el 2016 el Museu d'Arquelogia de Catalunya cuenta con la exposición temporal itinerante A la recerca dels tresors perduts (Rubens Productions), dedicada a la figura de este arqueólogo y sus películas.

No obstante, se trata de un fenómeno retroalimentado, ya que no es casual que el éxito de este tipo de películas fuese en los años 80 y 90, momento en el que la Arqueología se consolidó como profesión y cuyos resultados tenían ya un radio de difusión muy amplio. El cine siempre refleja la sociedad del momento en el que se produce (Aumont et al., 1985, 98). Esa sociedad mostraba ya fervor por el conocimiento de las culturas del pasado y, al mismo tiempo, tenía una mayor concienciación por la protección de los bienes que integraban ese patrimonio (por citar un caso cercano, durante los años 80 y 90 en España se promulgaron las leyes de patrimonio estatal y autonómicas). Eran los años en los que se comenzaban a desarrollar propuestas arqueológicas como claros productos culturales y turísticos (Ballart, 1997). Indy, al igual que las piezas que persigue, también es producto de su tiempo. 


\section{BIBLIOGRAFÍA}

ARANGO, C. A. (2008): "Indiana Jones o la pregunta por las peripecias de la aventura: Apuntes para la arqueología de un imaginario heroico", Revista Luciérnaga Audiovisual, 1, 16-28.

AUMONT, J., BERGALA, A., MARIE, M., VERNET, M. (1985): Estética del cine: espacio fílmico, montaje, narración, lenguaje, Barcelona, Paidós.

AZCONA, J. M., AVILÉS, J. (2013): “Aproximación a la historia contemporánea en el cine de Steven Spielberg”, Ferrol Análisis: Revista de pensamiento y cultura, 28, 149-162.

BALLART, J. (1997): El patrimonio histórico y arqueológico: valor y uso, Barcelona, Ariel.

BARCELÓ, C. (1998): La Atlántida, Madrid, M.E.

BONN-MULLER, E., POWELL, E. A. (2008): "Indy Spirit Awards”, Archaeology, 61, 3 (https://archive.archaeology.org/0805/trenches/indy.html, consultado 01-03-2018).

BORDWELL, D. (1996): La narración en el cine de ficción, Barcelona, Paidós.

BRISSAUD, P., ZIVIE-COCHE, C. (2000): Tanis: travaux récents sur le tell Sân elHagar. 1997-2000, París, Noêsis.

CANO, E. (2017): Indiana Jones: Las Reliquias Desveladas, Madrid, Atlantis.

CARVAJAL, A., HERNANDO, C., DE SOTO, M. R., TEJERIZO, C. (2011): “El síndrome de Indiana Jones. La imagen social del arqueólogo", Estrat Crític, 5, vol. 3 , 38-49.

DE LA PLAZA, L., MARTÍNEZ MURILLO, J. M., VAQUERO, J. I. (2016): Guía para identificar los personajes de la mitología clásica, Madrid, Cátedra.

DURÁn, M. A., LISI, F. (1992). Platón. Diálogos VI. Filebo, Timeo, Critias, Traducciones, introducciones y notas, Madrid, Gredos.

FORSSMANN, A. (2017): "Nuevos hallazgos frente a la costa siciliana: 47 lingotes de oricalco y 2 cascos corintios", National Geographic España (http://www.nationalgeographic.com.es/historia/actualidad/nuevos-hallazgos-frente costa-sicilianalingotes-oricalco-cascos-corintios_11169, consultado 01-04-2018).

GONZÁLEZ, R. (2011): "En busca de Indiana Jones", Revista de Arqueología, 365, 52-63.

GRESH, L. H., WEINBERG, R. (2008): ¿Por qué tenían que ser serpientes? Los misterios de Indiana Jones, Barcelona, Robin Book.

HALL, M. A. (2004): "Romancing the stones: Archaeology in popular cinema", European Journal of Archaeology, 7 (2), 159-176.

HÄRKE, H. (2014): “Archaeology and Nazism: A Warning from Prehistory” en Mordvintseva, V., Härke, H., Shevchenko, T. (eds.), Archaeological and linguistic research: Materials of the Humboldt-Conference (Simferopol-Yalta, 20-23 September, 2012), Kiev, 32-42.

LIVERANI, M. (2005): Más allá de la Biblia. Historia Antigua de Israel, Barcelona, Crítica. 
LUCENO, J. (2008): Indiana Jones. Guía Visual, Madrid, SM.

MARINATOS, S. (1939): "The volcanic destruction of Crete", Antiquity, XIII, 425-439.

MARINATOS, N. (2015): Akrotiri. Thera and the East Mediterranean, Atenas, Militos.

MITCHELL-HEDGES, F. A. (1954): Danger My Ally, Londres, Adventures Unlimited Press.

RAHN, O. W. (1982): Cruzada contra el Grial: la tragedia del catarismo, Madrid, Hiperion.

RUÍZ ZAPATERO, G., FERNÁNDEZ MARTÍNEZ, V. M. (2008): “Arqueología: Imagen y proyección social", Complutum, 8, 263-264.

SZCZEPAŃSKI, S. (2009): “Archaeology in the service of the nazis: Himmler's propaganda and the excavations at the hillfort site in Stary Dzierzgon (Alt Christburg)", Lietuvos Archeologija, 35, 83-94.

SHOHAT, E., STAM, R. (1994): Unthinking Eurocentrism, Multiculturalism and the Media, London, Psychology Press.

VAN WOERKENS, M. (2002): The Strangled Traveler: Colonial Imaginings and the Thugs of India, Chicago, University of Chicago Press.

VIZCAINO, A. (2014): "El mirall de Lara Croft: arqueòlogues en un món de ficció", Saguntum - PLAV Extra 15, 17-24.

VVAA (2008): Indiana Jones. Todo sobre la saga, Especial Fotogramas Coleccionistas, Barcelona.

WALSH, J. M. (2005): "What is real? A new look at Precolumbian Mesoamerican Collections", AnthroNotes: Museum of Natural History Publication for Educators, 26 (1), 1-7 y 17-19.

WALSH, J. M. (2008): "Legend of the Crystal Skulls“, Archaeology, 61 (3), 36,41. https://archive.archaeology.org/0805/etc/indy.html (consultado 01-03-2018).

WALSH, J. M. (2010): “The Skull of Doom”, Archaeology, online features. archive.archaeology.org/online/features/mitchell_hedges/ (consultado 01-03-2018).

\section{FILMOGRAFÍA}

BENNETT, C., MARTON, A. (1950): King Solomon's Mines (Las minas del rey Salomón).

DE BONT, J. (2003): Lara Croft Tomb Raider: The Cradle of Life (Lara Croft Tomb Raider: La cuna de la vida).

GATO, E. (2012): Las aventuras de Tadeo Jones.

GATO, E., ALONSO, D. (2017): Tadeo Jones 2: El secreto del rey Midas.

HOPPER, J. (1954): The Secret of the Incas (El secreto de los incas).

HUSTON, J. (1948): The Treasure of the Sierra Madre (El tesoro de Sierra Madre). LEE THOMPSON, J. (1985): King Solomon's Mines (Las minas del rey Salomón).

PIROSH, R. (1954): Valley of the Kings (El Valle de los Reyes).

SOMMERS, S. (1999): The Mummy (La Momia).

SOMMERS, S. (2001): The Mummy Returns (El retorno de la Momia). 
SPIELBERG, S. (1981): Raiders of the Lost Ark (En busca del arca perdida).

SPIELBERG, S. (1984) Indiana Jones and the Temple of Doom (Indiana Jones y el templo maldito).

SPIELBERG, S. (1989) Indiana Jones and the Last Crusade (Indiana Jones y la última cruzada).

SPIELBERG, S. (2008): Indiana Jones and the Kingdom of the Crystal Skull.

STEVENS, G. (1939): Gunga Din.

TURTELTAUB, J. (2004): National Treasure (La búsqueda).

UTHAUG, R. (2018): Tomb Raider.

WEST, S. (2001): Lara Croft: Tomb Raider.

ZEMECKIS, R. (1984): Romancing the Stone (Tras el corazón verde). 